\title{
Clinical practice pattern of low back pain among physiotherapists in a low-income country
}

\author{
Mohammad Ali ${ }^{1}$, Zakir Uddin ${ }^{2}$, and Ahmed Hossain ${ }^{3}$ \\ ${ }^{1}$ Uttara Adhunik Medical College \\ ${ }^{2}$ McMaster University Faculty of Health Sciences \\ ${ }^{3}$ North South University
}

August 3, 2020

\begin{abstract}
Background: Low back pain (LBP) is the top global cause of disability and physiotherapy interventions are used to manage it. However, the practice pattern of physiotherapists dealing with LBP patients in low-income countries are limited. Aim: The study aims to explore the LBP practice pattern of a low-income country's ( i. e., Bangladeshi) physiotherapists by their demographic and professional factors. Methods: In a cross-sectional survey study, we have analyzed data from randomly selected 423 physiotherapists of Bangladesh who have invited to fill-up an online survey questionnaire about practice patterns. The first part of the questionnaire contained question demographic and professional background, second part included current intervention choices in the management of patients with LBP, the final part consisted of information on diagnosis, patient type and selfreported cure rate of LBP patients. Ethical approval: Clinical Trial Registry India: CTRI/2020/05/025313. Results: The Majority of the physiotherapists (54.8\%) were non-government service holders and $87.7 \%$ worked in the town area. Regarding recommended interventions, only $12.3 \%$ frequently used those and $21.5 \%$ didn't either offer or know about those interventions. For not recommended interventions, $69.3 \%$ occasionally, $13.5 \%$ frequently and $17.3 \%$ never used such interventions. The prevalence of good, moderate, and poor practice patterns was $14 \%, 62.4 \%$, and $23.6 \%$ respectively. Participants' marital status $(\mathrm{P}=0.003)$ and graduation institute category $(\mathrm{P}=0.002)$ were significant factors for practice pattern variation. Conclusion: The study justified physiotherapy management status in a low-income country by comparing evidence-based practice guidelines. This finding set as a low-income country database to exhibit future research, clinical practice, and education for better LBP physiotherapy management adherence to evidence-based public health care.
\end{abstract}

\section{Clinical practice pattern of low back pain among physiotherapists in a low-income country}

Running title: Low back pain practice pattern of Physiotherapist

Mohammad Ali ${ }^{*}$, Zakir Uddin², Ahmed Hossain ${ }^{3}$

${ }^{1}$ Department of Physiotherapy and Rehabilitation, Uttara Adhunik Medical College and Hospital, Dhaka1230, Bangladesh.

${ }^{2}$ School of Rehabilitation Sciences, McMaster University, Hamilton, Ontario-L8S 4L8, Canada.

${ }^{3}$ Department of Public Health, North South University, Bashundhara, Dhaka-1229, Bangladesh.

* Corresponding author: Mohammad Ali, E-mail: alibup2018@gmail.com

Emails: Mohammad Ali: alibup2018@gmail.com

Zakir Uddin: uddinz2@mcmaster.ca

Ahmed Hossain: ahmed.hossain@utoronto.ca 


\title{
ORCID id:
}

Mohammad Ali: 0000-0002-4685-5050

Zakir Uddin: 0000-0002-3018-0327

Ahmed Hossain: 0000-0003-4575-1273

\section{Abstract}

\section{Background:}

Low back pain (LBP) is the top global cause of disability and physiotherapy interventions are used to manage it. However, the practice pattern of physiotherapists dealing with LBP patients in low-income countries are limited.

\begin{abstract}
Aim:
The study aims to explore the LBP practice pattern of a low-income country's (i. e., Bangladeshi) physiotherapists by their demographic and professional factors.

Methods:

In a cross-sectional survey study, we have analyzed data from randomly selected 423 physiotherapists of Bangladesh who have invited to fill-up an online survey questionnaire about practice patterns. The first part of the questionnaire contained question demographic and professional background, second part included current intervention choices in the management of patients with LBP, the final part consisted of information on diagnosis, patient type and self-reported cure rate of LBP patients.
\end{abstract}

\section{Ethical approval:}

Clinical Trial Registry India: CTRI/2020/05/025313.

\section{Results:}

The Majority of the physiotherapists $(54.8 \%)$ were non-government service holders and $87.7 \%$ worked in the town area. Regarding recommended interventions, only $12.3 \%$ frequently used those and $21.5 \%$ didn't either offer or know about those interventions. For not recommended interventions, $69.3 \%$ occasionally, $13.5 \%$ frequently and $17.3 \%$ never used such interventions. The prevalence of good, moderate, and poor practice patterns was $14 \%, 62.4 \%$, and $23.6 \%$ respectively. Participants' marital status $(\mathrm{P}=0.003)$ and graduation institute category $(\mathrm{P}=0.002)$ were significant factors for practice pattern variation.

\section{Conclusion:}

The study justified physiotherapy management status in a low-income country by comparing evidence-based practice guidelines. This finding set as a low-income country database to exhibit future research, clinical practice, and education for better LBP physiotherapy management adherence to evidence-based public health care.

Keyword: Low back pain, physiotherapy, practice pattern

\section{Introduction}

Low back pain (LBP) is the top global cause of disability ${ }^{1}$ and the incidence rate varies from $0.024-7.0 \%{ }^{2}$. The prevalence of LBP is higher among the population groups with low socioeconomic status ${ }^{3,4}$. Data indicated that middle and low-income countries in Asia, Africa, and the Middle East are becoming the epicenter of LBP related disabilities due to an increased number of aged populations and poor health systems in these regions ${ }^{5}$. Previous studies found that the poor referral system, less availability of essential services in the rural areas, lack of proper guidelines for LBP intervention made the situation worst especially in the Indian subcontinent ${ }^{6,7}$. There is a tremendous opportunity to reduce the gap between existing and efficient intervention system for patients in low-income countries by identifying the improvement opportunities. 
There are a plethora of surgical, pharmacological, and non-pharmacological treatment options for LBP whereas, very few of them are effective to reduce LBP burden ${ }^{8,9}$. Physiotherapy is an effective treatment option for LBP, but all the interventions are used in this method are not equally beneficial ${ }^{10,11}$. Frequently used modalities for LBP in low and middle-income countries such as short wave diathermy, ultrasound, interferential therapy, transcutaneous electric stimulation, traction, and back support ${ }^{6,12,13}$ are found ineffective and not recommended ${ }^{14-17}$. Guidelines recommended mainly cognitive behavioral therapy, progressive relaxation, and mindfulness-based stress reduction and combined packages of physical and psychological intervention for LBP ${ }^{14-16}$. However, a systematic review and meta-analysis in 2019 concluded that the rate of interventions provided by the physiotherapist for LBP that were $35 \%$ recommended, $44 \%$ not recommended, and $72 \%$ had no recommendations ${ }^{18}$. Nonetheless, studies included in this review mostly were from highincome countries. Thus very few are known about the current practice pattern of the physiotherapist for LBP in low-income countries such as Bangladesh.

Bangladesh is the $8^{\text {th }}$ most populous and $12^{\text {th }}$ densely populated country in the world with 160 million people 19. Unsurprisingly, there is a substantial difference between the numbers of physiotherapists for per million people in high-income and middle or low-income countries. In contrast with 209 thousand and 52 thousand registered physiotherapist in the US and UK for 329 million and 65 million people respectively ${ }^{20,21}$, there are currently only 1.7 thousand registered physiotherapists for 160 million people in Bangladesh ${ }^{22}$. On the

other hand, previous studies found a high prevalence of LBP among different groups of the population in Bangladesh $^{23-26}$. To ensure quality management by utilizing limited resources for a large number of LBP patients in Bangladesh, exploring the practice pattern of the treatment provider is warranted. Furthermore, to make a promising guideline of a country to improve health-care outcomes and potentially reduce costs by effectively implementing known best practice recommendations, we must need to know the practice pattern of physiotherapists' dealing with LBP patients in that particular country. The study aims to explore the LBP practice pattern of Bangladeshi Physiotherapists considering their demographic and professional factors.

\section{Methods}

\subsection{Design and materials}

A cross-sectional descriptive survey with a self-administered questionnaire was conducted using the STROBE cross-sectional reporting guidelines ${ }^{27}$ from May 23 to June 6, 2020. The format of this questionnaire was proved successful in previous studies of physiotherapy management for LBP ${ }^{6,13}$. Before distribution, the questionnaire was piloted with a convenient 20 Bangladeshi physiotherapists. These physiotherapists were asked to give their opinion regarding the questionnaire format, its content, wording, instructions, and ease of completion. The questionnaire was slightly revised as the feedback given.

The content of the questionnaire was divided into three parts. In the first part, the respondent was asked about their demographic and professional background. In the second part, physiotherapists were asked to provide details on their current intervention choices in the management of patients with LBP. The final part consisted of information about the use of x-ray for LBP diagnosis, patient type, and self-reported cure rate of LBP patients.

\subsection{Inclusion-exclusion criteria and ethical consideration}

Registered graduate physiotherapists who are currently living and practicing in Bangladesh, age 18-65 years, see a minimum of one LBP patient per day in their practice, willing to participate in this study were the inclusion criteria. We exclude who are registered in Bangladesh but currently living or practicing outside of Bangladesh, age more than 65 years. Voluntary informed consent was taken from all the participants.

We have taken prospective observational trial registration from the World Health Organization (WHO) endorsed Clinical Trial Registry- India (CTRI/2020/05/025313 [Registered on 22/05/2020]). The ethical review committee of Uttara Adhunik Medical College and Hospital has also approved the study.

\subsection{Sample size determination}


There were 1793 registered physiotherapists in Bangladesh at the time of our study commenced. Using finite population sample size formula ${ }^{28-30}$, we have calculated the minimum sample size 317 after considering a $95 \%$ confidence interval and 5\% marginal error. However, we invited 1000 randomly selected physiotherapists using digital communication tools (e.g. Email, Messenger) for participating in this study. 98 communication account was not either valid or active. However, 639 physiotherapists filled and returned the form (response rate of $70.80 \%$ ). After considering the inclusion and exclusion criteria, we found 423 data eligible for this study. We have put these final data for analysis in a password encrypted personal computer with a new unidentifiable code number after removing participants' names and registration digit to ensure confidentiality.

\subsection{Demographic and professional factors}

In this survey, demographic factors of the participant such as age, sex, and marital status were recorded. The physiotherapists were also asked to provide their professional factors (e.g. highest qualification, specialization, type of institute from where they were graduated, type of organization currently working, service type, working station, and patient load per day).

\subsection{Practice pattern}

Eighteen interventions were included in this study (list of the interventions are in Table 1). We evaluated practice patterns in two different ways based on these interventions' use frequency and evidence level.

Firstly, the respondents were asked to report the frequency of each intervention on a Likert scale from 1 to 3 ; with 1 indicating frequent use, 2 for occasional use and 3 indicating never use. We have also included additional 'unknown intervention' options for cognitive behavioral therapy, progressive relaxation, and mindfulness-based stress reduction, and interdisciplinary rehabilitation intervention. We sub-categories these 18 interventions into (i) not recommended (ii) partially recommended and (iii) recommended groups considering treatment guidelines for LBP ${ }^{14-17,31-33}$. The frequency and percentage of the physiotherapists who used these eighteen different intervention options are used as the first way to explain practice patterns.

Secondly, to segregate practice pattern to the poor, moderate and good; we calculated points in the following way: (a) for not recommended interventions, physiotherapists were given 0 points for selecting frequent use, 1 point for occasional use, 2 points for never use; (b) for partially recommended interventions, never use selectors were given 0 points, while occasional and frequent selectors were given 1 point; (c) for recommended interventions, 0 for never use and unknown intervention, 1 for occasional use and 2 for frequent use. The total point was ranging from 0-28. The median, first quartile, and the third quartile of the points were 15 , 13, and 17 respectively. Participants who scored equal and/or above the third quartile were categorized as a "good practitioner", equal and/or below the first quartile were separated as a "poor practitioner" and finally, the physiotherapist who scored between first and third quartile was identified as a "medium practitioner" in this study.

\subsection{Data analysis}

To analyze the data, we use Statistical Package for the Social Science (SPSS) software version 20.0, SPSS Inc., Chicago, IL, USA. Descriptive statistical analysis was done to find the physiotherapists' frequency and percentage for different categories and the different interventions. When testing the differences between groups, $\mathrm{P}$-value is calculated from the chi-square test. A significance level of $\mathrm{p}<0.05$ was used.

\section{Result}

\subsection{Demographic and professional factors}

Table 2 shows the demographic and professional factors of physiotherapists in Bangladesh. Participants were dominated by young age groups where $66 \%$ were from $26-35$ years' age group. $76.8 \%$ of participants were male while the percentage of graduate and post-graduate physiotherapists were the same $(49.2 \%$ vs $50.8 \%$ ). $44.7 \%$ physiotherapists were specialized in musculoskeletal physiotherapy followed by no specialization group (28.1\%). $63.6 \%$ physiotherapists have been graduated from the private institute and $31.2 \%$ worked in general hospital/clinic followed by $30.7 \%$ and $26.7 \%$ worked in rehabilitation institutes and private 
chambers respectively. More than half of the physiotherapists (54.8\%) were non-government service holder and $32.9 \%$ are self-employed. Among all the participants, $87.7 \%$ worked in town and the majority $(67.1 \%)$ have the patient load in between 1-5 patients per day.

\subsection{Practice pattern}

Firstly, we calculate the frequency and percentage of a physiotherapist who used eighteen different intervention options (in table 1). Among all eighteen interventions, the highest $92.4 \%$ physiotherapist frequently used exercise therapy, $83.2 \%$ occasionally used mechanical traction and $66.9 \%$ never used acupuncture, while the highest $37.6 \%$ participants said that Mindfulness-based stress reduction therapy was unknown to them. Figure 1 shows the average number of participants used three groups of interventions according to the frequency of use (frequently, occasionally, never). On average, 293 participants (69.3\% of total) occasionally used not recommended intervention, 57 (13.5\% of total) frequently and only 73 (17.3\% of total) participants never used such intervention. For the partially recommended group, the average number of participants for frequent, occasional, and never used intervention was 141 (33.3\% of total), 185 (43.7\% of total), and 97 ( $23 \%$ of total) respectively. Nonetheless, only 52 (12.3\% of total) participants frequently used recommended intervention on an average. One the other hand, on an average, 91 (21.5\% of total) physiotherapist either do not offer or do not know about the recommended interventions.

Secondly, table 2 has shown the result of the analysis of good, moderate, and poor practice patterns after calculating given points to each physiotherapist for the choice of different group interventions. The prevalence of good practice among physiotherapist of Bangladesh were $14 \%$, however, $62.4 \%$ and $23.6 \%$ did moderate and poor practice respectively. A higher number of the younger group (18-25 years of age) of physiotherapists do good practice. Among all the demographic and professional factors in this study, only the marital status $(P=0.003)$ and graduation institute category $(P=0.002)$ were significant factors for practice pattern variation (Table 2 ).

In addition to 18 interventions, we evaluated a diagnostic (radiological imaging) dependency, LBP patient category has commonly seen, and cure rate patterns. We estimated the dependency of a physiotherapist on radiological imaging for LBP diagnosis and found $97.8 \%$ physiotherapists either frequently or occasionally use x-ray and/or MRI. For LBP patient category, $67.2 \%$ of participants said that they have seen mainly chronic LBP patients in their practice. When asked cure rate, $46.8 \%$ physiotherapists claimed that the cure rate of their LBP patients was $71-90 \%$ and $35.7 \%$ said this rate was between $51-70 \%$.

\section{Discussion}

We found a high prevalence of moderate practice habits and a low prevalence of good practice habits for LBP among the physiotherapist in Bangladesh. Though most of the currently practicing physiotherapists in Bangladesh were young, only less than one fourth was female. Half of the participants had a post-graduate degree and the impressive number of them had a specialization in musculoskeletal physiotherapy. However, data showed that there is a scarcity of physiotherapists in the government's health sector in Bangladesh and most of them had worked in the city area. Dependency on passive electrical and physical modalities and exercise therapy were very high among the physiotherapist in Bangladesh. Very few used recommended intervention for example Mindfulness-based stress reduction therapy for LBP. Unsurprisingly, for diagnosis of LBP, all most all the physiotherapists advise radiological imaging, though most of the respondents reported that they mainly have seen chronic LBP patients in their practice.

Little is known about current practice patter of physiotherapists in low and middle-income countries compared to high-income countries. A most recent study that seen the practice pattern of physiotherapists in the low-income country has been done in Ghana in 2013. Like our study, this study reported that most physiotherapists used not recommended and partially recommended interventions ${ }^{34}$. In our study, we have found $92.4 \%$ of physiotherapists offer exercise therapy for LBP. However, this rate was $100 \%$ in Ghana, although most of our participants were young and less experienced like Ghana. This indicated that physiotherapy is a relatively newer profession for low-income countries. However, a study conducted in 2010 in India, found the same picture as our study regarding the use of electrotherapy and exercise therapy ${ }^{6}$. Previous studies from 
Thailand (in 2005) and Nigeria (in 2007) reflected the same scenario ${ }^{12,13}$. It means the practice pattern of physiotherapists in low and middle-income countries is almost the same over the one and half-decade. More studies from other low and middle-income countries can give us a recent practice pattern of physiotherapists. For an example of data from high-income countries, a systematic review estimated $50 \%$ of physiotherapists used not recommended interventions for $\mathrm{LBP}^{18}$. However, homogenous studies from low and middle-income countries are not yet enough for making a valid conclusion on practice pattern, more specifically for LBP.

In our study, we found half of the physiotherapists have masters and above degrees, and most of them are specialized in musculoskeletal physiotherapy, however, very few of them are a good practitioner. This indicates the necessity of revision of postgraduate courses in Bangladesh. It was impressive that the prevalence of good practice among young physiotherapist was higher than the aged. It is probably that the young generation is more connected with the outer world and conscious of modern treatment techniques. One the other hand, our study shows a higher number of physiotherapists who was graduated from a private institute and worked in the non-government institute were engaged in good practice. This finding is similar to the findings of other studies conducted in Bangladesh where the author found governments' impassivity about the physiotherapy profession in Bangladesh ${ }^{22}$.

Imaging has a very limited role in LBP management and unnecessary lumbar imaging is a harmful exposure to radiation. Imaging rates for LBP diagnosis are high all over the world. For example, $53.7 \%$ and $56 \%$ of patients with LBP are referred for imaging by general practitioners in the USA and Italy respectively ${ }^{35,36}$. Surprisingly, this rate is up to $100 \%$ in India and China ${ }^{37,38}$. In our study, we found $97.8 \%$ physiotherapist refer their patients for radiological imaging to diagnose LBP which is a clear waste of health-care resources in Bangladesh.

In our study, data have shown that Bangladeshi physiotherapist mainly relied on not recommended and partially recommended interventions. However, four out of five physiotherapists claim that the cure rate of their LBP patients is between 50-90\%. Nonetheless, two systematic reviews and meta-analysis suggested that only those patients who received evidence-based physiotherapy were satisfied and feel confident about physiotherapy interventions ${ }^{39,40}$. A large scale cohort study addressing the effect of physiotherapy on LBP patients in Bangladesh is needed to find the actual efficacy rate.

The first study in Bangladesh that evaluated the LBP practice pattern of an important professional group who are dealing with LBP predominantly. The study is focused on the top global cause of disability (i.e. LBP) and justified its management status (compared to evidence-based practice guidelines) in a low-income country. The response rate for this study was considerably high (70.8\%) and a good number of representatives from the city and rural area appropriately reflect the national situation for Bangladesh. Despite this, limitations such as the measuring bias in self-reported data ${ }^{41}$ should be recognized. The current data were based on participants' freedom opinion and relied on their memory, which might oppose to actual intervention pattern of patients. The difference in interpretation might lead due to the use of predominantly closed questions. Future research with the combination of quantitative and qualitative data can overcome the limitation. Another important limitation of this study is that it does not characterize between cases of acute and chronic LBP according to guideline recommendation. For example, cognitive behavioral therapy is recommended for chronic low back pain but not for acute low back pain. However, the majority of the participant said that they mainly treat chronic LBP patients in their practice.

Our findings help advance the scientific literature in this area as an example of professional practice pattern evidence from a low-income country. This study has valuable implications for the management of LBP by the physiotherapist, especially in a low-income country like Bangladesh. The findings set as a database to exhibit future research, clinical practice, and education for the physiotherapy profession. Although, future clinical trials focusing on the specific evidence-based application of interventions used by Bangladeshi physiotherapists are needed to provide acuteness into the outcome of LBP management in Bangladesh. The current practice pattern is known from this study, which is a baseline reference point for further upgrades, and it's helpful to reduce the burden of disability by improving LBP physiotherapy management adherence to evidence-based public health care. 


\section{References}

1. Vos T, Abajobir AA, Abate KH, et al. Global, regional, and national incidence, prevalence, and years lived with disability for 328 diseases and injuries for 195 countries, 1990-2016: a systematic analysis for the Global Burden of Disease Study 2016. Lancet . 2017;390(10100):1211-1259. doi:10.1016/S0140-6736(17)32154-2

2. Fatoye F, Gebrye T, Odeyemi I. Real-world incidence and prevalence of low back pain using routinely collected data. Rheumatol Int . 2019;39(4):619-626. doi:10.1007/s00296-019-04273-0

3. Ikeda T, Sugiyama K, Aida J, et al. Socioeconomic inequalities in low back pain among older people: The JAGES cross-sectional study. Int J Equity Health . 2019;18(1):15. doi:10.1186/s12939-019-0918-1

4. Suman A, Bostick GP, Schaafsma FG, Anema JR, Gross DP. Associations between measures of socioeconomic status, beliefs about back pain, and exposure to a mass media campaign to improve back beliefs. BMC Public Health . 2017;17(1):504. doi:10.1186/s12889-017-4387-4

5. Hartvigsen J, Hancock MJ, Kongsted A, et al. What low back pain is and why we need to pay attention. Lancet . 2018;391(10137):2356-2367. doi:10.1016/S0140-6736(18)30480-X

6. Fidvi N, May S. Physiotherapy management of low back pain in India - a survey of self-reported practice. Physiother Res Int . 2010;15(3):150-159. doi:10.1002/pri.458

7. Bashar M, Bhattacharya S, Tripathi S, Sharma N, Singh A. Strengthening primary health care through e-referral system. J Fam Med Prim Care . 2019;8(4):1511. doi:10.4103/jfmpc.jfmpc_184_19

8. Foster NE. Barriers and progress in the treatment of low back pain.BMC Med . 2011;9:108. doi:10.1186/1741-7015-9-108

9. Pransky G, Buchbinder R, Hayden J. Contemporary low back pain research - and implications for practice. Best Pract Res Clin Rheumatol . 2010;24(2):291-298. doi:10.1016/j.berh.2010.01.001

10. Shipton EA. Physical Therapy Approaches in the Treatment of Low Back Pain. Pain Ther . 2018;7(2):127-137. doi:10.1007/s40122-018-0105-x

11. Foster NE, Anema JR, Cherkin D, et al. Prevention and treatment of low back pain : evidence , challenges , and promising directions. 2018;391. doi:10.1016/S0140-6736(18)30489-6

12. Ayanniyi O, Lasisi O., Adegoke BO., Oni-Orisan M. Management of low back pain: - attitudes and treatment preferences of physiotherapists in Nigeria. African J Biomed Res . 2009;10(1):41-49. doi:10.4314/ajbr.v10i1.48970

13. Pensri P, Foster NE, Srisuk S, Baxter GD, McDonough SM. Physiotherapy management of low back pain in Thailand: a study of practice. Physiother Res Int . 2005;10(4):201-212. doi:10.1002/pri.16

14. Stochkendahl MJ, Kjaer P, Hartvigsen J, et al. National Clinical Guidelines for non-surgical treatment of patients with recent onset low back pain or lumbar radiculopathy. Eur Spine J . 2018;27(1):60-75. doi:10.1007/s00586-017-5099-2

15. Qaseem A, Wilt TJ, McLean RM, Forciea MA. Noninvasive Treatments for Acute, Subacute, and Chronic Low Back Pain: A Clinical Practice Guideline From the American College of Physicians. Ann Intern Med . 2017;166(7):514. doi:10.7326/M16-2367

16. NICE. Recommendations | Low Back Pain and Sciatica in over 16s: Assessment and Management | Guidance | NICE . NICE; 2016. https://www.nice.org.uk/guidance/ng59/chapter/Recommendations. Accessed June 6, 2020.

17. Godfrey CM. Physical therapy in the treatment of low back pain.Appl Ther . 1966;8(11):950-953. doi:10.6084/m9.figshare.7039769 
18. Zadro J, O'Keeffe M, Maher C. Do physical therapists follow evidence-based guidelines when managing musculoskeletal conditions? Systematic review. BMJ Open . 2019;9(10):e032329. doi:10.1136/bmjopen2019-032329

19. The World Bank. Population, total - Bangladesh | Data. The World Bank. https://data.worldbank.org/indicator/SP.POP.TOTL?locations=BD. Published 2019. Accessed June 7, 2020 .

20. Chartered Society of Physiotherapy | World Confederation for Physical Therapy. https://www.wcpt.org/node/25749. Accessed June 7, 2020.

21. Brightfind B. About Physical Therapist (PT) Careers. American Physical Therapy Association. http://www.apta.org/PTCareers/Overview/. Published 2016. Accessed June 7, 2020.

22. Mamin FA, Hayes R. Physiotherapy in Bangladesh: Inequality Begets Inequality. Front Public Heal . 2018;6. doi:10.3389/fpubh.2018.00080

23. Hossain MD, Aftab A, Al Imam MH, et al. Prevalence of work related musculoskeletal disorders (WMSDs) and ergonomic risk assessment among readymade garment workers of Bangladesh: A cross sectional study. Guo NL, ed. PLoS One . 2018;13(7):e0200122. doi:10.1371/journal.pone.0200122

24. Sanjoy SS, Ahsan GU, Nabi H, Joy ZF, Hossain A. Occupational factors and low back pain: a crosssectional study of Bangladeshi female nurses.BMC Res Notes . 2017;10(1):1-6. doi:10.1186/s13104-017-24921

25. Hossain A. Prevalence and Occupational Factors Associated with Low Back Pain Among the Female Garment Workers: A Cross-Sectional Study in Bangladesh. Glob Perspect Med Sci . 2018;2(1):8. doi:10.26535/gpms.v2i1.10

26. Mohammad Ali, Gias U. Ahsan and AH. Prevalence and associated occupational factors for low back pain among the bank employees in Dhaka City. medRxiv . 2019. https://www.medrxiv.org/content/10.1101/19012328v1.full.pdf+html.

27. von Elm E, Altman DG, Egger M, Pocock SJ, Gøtzsche PC, Vandenbroucke JP. The Strengthening the Reporting of Observational Studies in Epidemiology (STROBE) statement: guidelines for reporting observational studies. J Clin Epidemiol . 2008;61(4):344-349. doi:10.1016/j.jclinepi.2007.11.008

28. Singh, Ajay S, Masuku MB. Sampling Techniques \& Dertermning Sample Size in Applied Statistics Research: an Overview. Interanational J Econ Commer Manag . 2014;II(11):1-22. http://ijecm.co.uk/. Accessed June 2, 2020.

29. Conroy R. Sample Size: introduction 1 Sample size A rough guide How to use this guide. 2006:9-12. https://pdfs.semanticscholar.org/4781/878153e13322c028c7d8970e7f52fbaa102a.pdf. Accessed June 2, 2020.

30. Fosgate GT. Practical Sample Size Calculations for Surveillance and Diagnostic Investigations. $J$ Vet Diagnostic Investig . 2009;21(1):3-14. doi:10.1177/104063870902100102

31. Hagen KB, Hilde G, Jamtvedt G, Winnem M. Bed rest for acute low-back pain and sciatica. In: Hagen KB, ed. Cochrane Database of Systematic Reviews . Chichester, UK: John Wiley \& Sons, Ltd; 2004. doi:10.1002/14651858.CD001254.pub2

32. Machado GC, Maher CG, Ferreira PH, et al. Efficacy and safety of paracetamol for spinal pain and osteoarthritis: systematic review and meta-analysis of randomised placebo controlled trials. BMJ . 2015;350(mar31 2):h1225-h1225. doi:10.1136/bmj.h1225

33. Foster NE, Anema JR, Cherkin D, et al. Prevention and treatment of low back pain: evidence, challenges, and promising directions.Lancet . 2018;391(10137):2368-2383. doi:10.1016/S0140-6736(18)30489-6 
34. Oppong-Yeboah B, May S. Management of Low Back Pain in Ghana: A Survey of Self-reported Practice. Physiother Res Int . 2014;19(4):222-230. doi:10.1002/pri.1586

35. Miceli L, Rizzardo A, Bednarova R, Guadagnin GM, Sbrojavacca R, Della Rocca G. Low-back pain at the emergency department: still not being managed? Ther Clin Risk Manag . 2016;12:183. doi:10.2147/TCRM.S91898

36. Rosenberg A, Agiro A, Gottlieb M, et al. Early Trends Among Seven Recommendations From the Choosing Wisely Campaign. JAMA Intern Med . 2015;175(12):1913. doi:10.1001/jamainternmed.2015.5441

37. Yu L, Wang X, Lin X, Wang Y. The Use of Lumbar Spine Magnetic Resonance Imaging in Eastern China: Appropriateness and Related Factors. Luo X, ed. PLoS One . 2016;11(1):e0146369. doi:10.1371/journal.pone.0146369

38. Sahu R. Non-drug non-invasive treatment in the management of low back pain. Ann Med Health Sci Res . 2014;4(5):780. doi:10.4103/2141-9248.141565

39. Chou L, Ranger TA, Peiris W, et al. Patients' perceived needs for allied health, and complementary and alternative medicines for low back pain: A systematic scoping review. Heal Expect . 2018;21(5):824-847. doi:10.1111/hex.12676

40. Lim YZ, Chou L, Au RT, et al. People with low back pain want clear, consistent and personalised information on prognosis, treatment options and self-management strategies: a systematic review. J Physiother . 2019;65(3):124-135. doi:10.1016/j.jphys.2019.05.010

41. Rosenman R, Tennekoon V, Hill LG. Measuring bias in self-reported data. Int $J$ Behav Healthc Res . 2011;2(4):320. doi:10.1504/IJBHR.2011.043414

42. Wong JJ, Côté P, Sutton DA, et al. Clinical practice guidelines for the noninvasive management of low back pain: A systematic review by the Ontario Protocol for Traffic Injury Management (OPTIMa) Collaboration.Eur J Pain . 2017;21(2):201-216. doi:10.1002/ejp.931

43. Steffens D, Maher CG, Pereira LSM, et al. Prevention of Low Back Pain. JAMA Intern Med . 2016;176(2):199. doi:10.1001/jamainternmed.2015.7431

44. Michaleff ZA, Kamper SJ, Maher CG, Evans R, Broderick C, Henschke N. Low back pain in children and adolescents: a systematic review and meta-analysis evaluating the effectiveness of conservative interventions. Eur Spine J . 2014;23(10):2046-2058. doi:10.1007/s00586-014-3461-1

Acknowledgments

All the authors acknowledge the participants for providing us the information to conduct the study. Authors also thank Bangladesh Physiotherapy Association and Bangladesh Physical Therapy Association for helping in data collection.

\section{Discloser}

Consent to Publish: Not applicable.

Availability of data

Data are available upon reasonable request. The data sets used and analyzed during the current study are available from the corresponding author on reasonable request.

Conflict of interest

The authors declare that they have no conflict of interests.

Funding

The study was not funded. 
Author's contributions

MA participated in study conception, design, formal statistical analysis, and coordination of the manuscript. $\mathrm{ZU}$ and $\mathrm{AH}$ reviewed and helped to draft the manuscript. ZU supervised the study. All authors read and approved the final manuscript.

Table 1: Interventions offered by physiotherapists and their practice frequency distribution $(\mathrm{n}=423)$

\begin{tabular}{|c|c|c|}
\hline List of interventions & Frequency of use (n, \%) & Frequency of use (r \\
\hline & Frequently & Occasionally \\
\hline Not recommended Intervention ${ }^{14-17,31,32}$ & Not recommended Intervention ${ }^{14-17,31,32}$ & Not recommend \\
\hline Short wave diathermy & $22(5.2)$ & $254(60.0)$ \\
\hline Ultrasound therapy & $70(16.5)$ & $269(63.6)$ \\
\hline Infrared radiation & $65(15.4)$ & $305(72.1)$ \\
\hline Transcutaneous electric nerve stimulation & $98(23.2)$ & $289(68.3)$ \\
\hline Mechanical traction & $25(5.9)$ & $352(83.2)$ \\
\hline Paracetamol & $12(2.8)$ & $298(70.4)$ \\
\hline Bed rest & $107(25.3)$ & $284(67.1)$ \\
\hline Partially recommended intervention ${ }^{33,42-44}$ & Partially recommended intervention ${ }^{33,42-44}$ & Partially recomn \\
\hline Exercise therapy & $391(92.4)$ & $30(7.1)$ \\
\hline Spinal manipulation & $88(20.8)$ & $305(72.1)$ \\
\hline Massage & $62(14.7)$ & $236(55.8)$ \\
\hline Acupuncture & $8(1.9)$ & $132(31.2)$ \\
\hline Yoga & $18(4.3)$ & $132(31.2)$ \\
\hline Superficial heat & $144(34.0)$ & $253(59.8)$ \\
\hline Postural education & $383(90.5)$ & $36(8.5)$ \\
\hline Back belt & $37(8.7)$ & $354(83.7)$ \\
\hline Recommended intervention ${ }^{14-16,33}$ & Recommended intervention ${ }^{14-16,33}$ & Recommended i \\
\hline Cognitive behavioral therapy & $47(11.1)$ & $192(45.4)$ \\
\hline Mindfulness-based stress reduction therapy & $30(7.1)$ & $174(41.1)$ \\
\hline Interdisciplinary rehabilitation & $78(18.4)$ & $203(48.0)$ \\
\hline
\end{tabular}

Table 2: Univariate analysis of demographic, professional factors and practice pattern $(\mathrm{n}=423)$

\begin{tabular}{llllll}
\hline Categories & Categories & $\mathrm{n}(\%)$ & Practice (n, Row \%) & Practice (n, Row \%) & Practice (n, \\
\hline \multirow{4}{*}{ Age } & & & Good & Moderate & Poor \\
& $18-25$ & $54(12.8)$ & $10(18.5)$ & $27(50.0)$ & $17(31.5)$ \\
\multirow{5}{*}{ Sex } & $26-35$ & $279(66.0)$ & $43(15.4)$ & $175(62.7)$ & $61(21.9)$ \\
\multirow{5}{*}{ Marital status } & $36+$ & $90(21.2)$ & $6(6.7)$ & $62(68.9)$ & $22(24.4)$ \\
\multirow{3}{*}{ Highest qualification } & Male & $325(76.8)$ & $43(13.2)$ & $201(61.8)$ & $81(25.0)$ \\
\multirow{3}{*}{ Specialization } & Female & $98(23.2)$ & $16(16.3)$ & $63(64.3)$ & $19(19.4)$ \\
& Unmarried & $153(36.2)$ & $31(20.3)$ & $81(52.9)$ & $41(26.8)$ \\
& Garried & $270(63.8)$ & $28(10.4)$ & $183(67.8)$ & $59(21.8)$ \\
& Post graduate & $208(49.2)$ & $33(15.9)$ & $120(57.7)$ & $55(26.4)$ \\
& Musculoskeletal & $215(50.8)$ & $26(12.1)$ & $144(67.0)$ & $45(20.9)$ \\
& Neurology & $189(44.7)$ & $30(15.9)$ & $117(61.9)$ & $42(22.2)$ \\
Graduation institute & $29(6.9)$ & $3(10.4)$ & $19(65.5)$ & $7(24.1)$ \\
& Pediatrics and gynecology & $23(5.5)$ & $5(21.7)$ & $17(74.0)$ & $1(4.3)$ \\
& Public health and others & $63(14.9)$ & $6(9.5)$ & $39(62.9)$ & $18(28.6)$ \\
& No specialization & $119(28.1)$ & $15(12.6)$ & $72(60.5)$ & $32(26.9)$ \\
& Public & $154(36.4)$ & $17(11.0)$ & $86(55.9)$ & $51(33.1)$
\end{tabular}




\begin{tabular}{llllll}
\hline Categories & Categories & $\mathrm{n}(\%)$ & Practice $(\mathrm{n}$, Row \%) & Practice (n, Row \%) & Practice $(\mathrm{n}$, \\
\hline \multirow{4}{*}{ Working institute } & Private & $269(63.6)$ & $42(15.6)$ & $178(66.2)$ & $49(18.2)$ \\
& Medical college hospital & $48(11.3)$ & $7(14.6)$ & $24(50.0)$ & $17(35.4)$ \\
& Rehabilitation institute & $130(30.7)$ & $24(18.5)$ & $83(63.8)$ & $23(17.7)$ \\
& General hospital/clinic & $132(31.2)$ & $15(11.4)$ & $89(67.4)$ & $28(21.2)$ \\
\multirow{5}{*}{ Service type } & Private chamber & $113(26.7)$ & $13(11.5)$ & $68(60.2)$ & $32(28.3)$ \\
& Government & $52(12.3)$ & $4(7.7)$ & $36(69.2)$ & $12(23.1)$ \\
\multirow{2}{*}{ Working station } & Non-government & $232(54.8)$ & $40(17.2)$ & $144(62.1)$ & $48(20.7)$ \\
& Self employed & $139(32.9)$ & $15(10.8)$ & $84(60.4)$ & $40(28.8)$ \\
Patient load per day & City & $371(87.7)$ & $53(14.3)$ & $230(62.0)$ & $88(23.7)$ \\
& Village & $52(12.3)$ & $6(11.5)$ & $34(65.4)$ & $12(23.1)$ \\
Total & 6-10 & $284(67.1)$ & $44(15.5)$ & $171(60.2)$ & $69(24.3)$ \\
& More than 10 & $88(20.8)$ & $11(12.5)$ & $55(62.5)$ & $22(25.0)$ \\
& Total & $51(12.1)$ & $4(7.8)$ & $38(74.5)$ & $9(17.6)$ \\
& & $423(100)$ & $59(14.0)$ & $264(62.4)$ & $100(23.6)$ \\
\hline
\end{tabular}

*P-value is calculated from the chi-square test. The significant values are bolded.

Figure 1: Practice pattern showing by the number of physiotherapists offering three categories of interventions and frequency of practice.

\section{Hosted file}

Fig_1_Clinical.docx available at https://authorea.com/users/348308/articles/473708-clinicalpractice-pattern-of-low-back-pain-among-physiotherapists-in-a-low-income-country 